\title{
Energy Coverage in Wireless Powered Sub-6 GHz and Millimeter Wave Dense Cellular Networks
}

\author{
Lifeng Wang, Kai-Kit Wong \\ Department of Electronic and Electrical Engineering, University College London, London, UK
}

\begin{abstract}
This paper focuses on the energy coverage in wireless powered sub-6 GHz and millimeter wave (mmWave) dense cellular networks, where mobile devices harvest RF energy from sub-6 GHz or mmWave base stations (BSs). The expressions for energy coverage probability in sub-6 GHz and mmWave tiers are respectively derived. The comparisons between sub-6 $\mathrm{GHz}$ and mmWave RF energy harvesting are analyzed. In particular, we provide the sufficient conditions for the case that wireless energy harvesting in mmWave tier is better than that in sub-6 GHz tier. Furthermore, in hybrid cellular networks with mode selection mechanism, the probability that a mobile device selects a sub-6 GHz BS or mmWave BS for wireless power transfer is also theoretically obtained.
\end{abstract}

\section{INTRODUCTION}

5G technologies such as massive multiple-input multipleoutput (MIMO) and millimeter wave (mmWave) make future cellular networks more suitable for wireless energy harvesting [1]. The very sharp signal beams in large-scale antenna systems are of great importance to wireless power transfer (WPT). In [2], the optimal power transfer beamforming was asymptotically derived by considering large number of antennas in a single massive MIMO cell, and the optimal solution for maximizing the throughput under the user fairness criterion was also asymptotically obtained. The work of [3] studied the effects of using large antenna arrays and dense small cells on WPT in the sub-6 GHz cellular networks, which showed that both technologies can substantially increase the amount of harvested energy. Recently, WPT in dense mmWave cellular networks with narrow beams has received much attention. Particularly, [4] first studied wireless powered mmWave cellular networks, where uniform linear array (ULA) with analog beamforming was implemented for wireless power transfer. In [5], downlink simultaneous wireless information and power transfer (SWIFT) was investigated in mmWave systems. The work of [6] further studied mmWave SWIFT in a more general way by considering both time switching and power splitting protocols. However, there are few results available for presenting WPT in the cellular networks encompassing sub- $6 \mathrm{GHz}$ cells and mmWave cells.

Motivated by the aforementioned, in this paper, we study wireless powered sub- $6 \mathrm{GHz}$ and mmWave dense cellular networks, in which sub-6 GHz or mmWave BSs can be selected to power mobile devices. A stochastic geometry approach is employed to characterize the BS density. We first derive the expressions for the energy coverage probability in sub- $6 \mathrm{GHz}$ and mmWave tiers. Based on the derived results, we provide conditions for the case that the amount of harvested energy in mmWave tier is larger than that in sub-6 GHz tier. In addition, we derive the connectivity probability that a mobile device selects a sub-6 GHz BS, line-of-sight (LoS) mmWave BS or a non-line-of-sight (NLoS) mmWave BS for power transfer. Simulation results have confirmed our analysis. The results show that the amount of harvested energy is dominated by directed power transfer, compared to the ambient RF energy harvesting. The amount harvested energy from ambient mmWave RF can still be larger than the sub- $6 \mathrm{GHz}$ counterpart in mmWave ultra-dense networks.

The notation of this paper is: $\dagger$ denotes the conjugate transpose operator, $\mathcal{C N}(\mathbf{0}, \boldsymbol{\Lambda})$ denotes the complex Gaussian distribution with zero mean and covariance matrix $\Lambda,\|\cdot\|$ denotes the Euclidean norm, $\mathbb{E}[\cdot]$ denotes the expectation operator, $\mathbf{0}_{M \times N}$ denotes the $M \times N$ zero matrix, $\mathbf{I}_{M}$ denotes the $M \times M$ identity matrix.

\section{SYSTEM DESCRIPTIONS}

\section{A. Network Model}

We consider a hybrid cellular network consisting of the sub-6 GHz small cells and mmWave small cells. Each sub$6 \mathrm{GHz}$ BS is equipped with $N$ sub-6 GHz antennas, and each mmWave BS is equipped with mmWave antenna array. The sub-6 GHz BSs are randomly located following a a homogeneous Poisson point process (HPPP) $\Phi_{\mu}\left(\lambda_{\mu}\right)$ with the density $\lambda_{\mu}$, and the mmWave BSs are randomly located following an independent HPPP $\Phi_{\mathrm{mm}}\left(\lambda_{\mathrm{mm}}\right)$ with the density $\lambda_{\mathrm{mm}}$. Particularly when devices requires the directed power transfer from a dedicated $\mathrm{BS}$, a sub- $6 \mathrm{GHz}$ mobile device $(\mu \mathrm{MD})$ will be connected with the sub- $6 \mathrm{GHz}$ BS that provides the largest received sub- $6 \mathrm{GHz}$ signal power, and accordingly, a mmWave mobile device (mmMD) will be connected with the mmWave BS that provides the largest received mmWave signal power. We assume that each $\mu \mathrm{MD}$ is equipped with a single sub- $6 \mathrm{GHz}$ antenna, and each mmMD is equipped with small mmWave antenna array, since the shorter mmWave wavelengths enable devices to pack more antennas for a given antenna aperture.

\section{B. Energy Harvesting in Sub-6 GHz Tier}

In sub-6 GHz tier, all the channels are assumed to undergo independent and identically distributed (i.i.d.) quasi-static Rayleigh block fading. Each sub-6 GHz BS uses maximumratio transmission (MRT) beamforming to transfer the energy for recharging its $\mu \mathrm{MD}$. Hence, for a typical $\mu \mathrm{MD} o$ connected with its serving sub-6 GHz BS, its instantaneous harvested 
power is written as

$$
\begin{aligned}
P_{r}^{\mu}= & \underbrace{\eta_{\mu} P_{\mu}\left\|\mathbf{h}_{o}\right\|^{2} L\left(\left|X_{o}\right|\right)}_{\Lambda_{\mu_{1}}} \\
& +\underbrace{\eta_{\mu} \sum_{k \in \Phi_{\mu}\left(\lambda_{\mu}\right) \backslash\{o\}} P_{\mu}\left|\mathbf{h}_{k, o} \frac{\mathbf{h}_{k}^{\dagger}}{\left\|\mathbf{h}_{k}\right\|}\right|^{2} L\left(\left|X_{k, \mu}\right|\right)}_{\Lambda_{\mu_{2}}},
\end{aligned}
$$

where $\Lambda_{\mu_{1}}$ is the directed transferred power, and $\Lambda_{\mu_{2}}$ is the total power from the ambient sub- $6 \mathrm{GHz}$ RF, $\eta_{\mu}$ is the sub- $6 \mathrm{GHz}$ RF-to-DC conversion efficiency, $P_{\mu}$ is the transmit power of sub-6 GHz BS, $\mathbf{h}_{o} \sim \mathcal{C N}\left(\mathbf{0}, \mathbf{I}_{N}\right)$ and $\left|X_{o}\right|$ are the small-scale fading channel vector and distance between the typical $\mu \mathrm{MD}$ and its serving $\mathrm{BS}$, respectively, $L\left(\left|X_{o}\right|\right)=\beta_{\mu}\left(\left|X_{o}\right|\right)^{-\alpha_{\mu}}$ is the pathloss function with the exponent $\alpha_{\mu}$, here, $\beta_{\mu}$ is the frequency dependent constant value, which is commonly set as $\left(\frac{\mathrm{c}}{4 \pi f_{c}}\right)^{2}$ with $c=3 \times 10^{8} \mathrm{~m} / \mathrm{s}$ and the carrier frequency $f_{c}$, $\frac{\mathbf{h}_{k}^{\dagger}}{\left\|\mathbf{h}_{k}\right\|}$ is the MRT beamforming vector of the sub-6 GHz BS $k$ $\left(k \in \Phi_{\mu} \backslash\{o\}\right)$ with $\mathbf{h}_{k} \sim \mathcal{C N}\left(\mathbf{0}, \mathbf{I}_{N}\right), \mathbf{h}_{k, o} \sim \mathcal{C N}\left(\mathbf{0}, \mathbf{I}_{N}\right)$ and $\left|X_{k, \mu}\right|$ are the small-scale fading channel vector and distance between the typical $\mu \mathrm{MD}$ and the sub- $6 \mathrm{GHz}$ BS $k$ (except the serving sub-6 GHz BS), respectively.

\section{Energy Harvesting in MmWave Tier}

In mmWave tier, the effect of small-scale fading can be negligible [7]. In addition, a sectored model is applied to analyze the beam pattern [8], i.e., the effective antenna gain for a mmWave $\operatorname{BS} \ell\left(\ell \in \Phi_{\mathrm{mm}} \backslash\{o\}\right)$ seen by the typical mmMD $o$ is expressed as

$$
G_{\ell}= \begin{cases}\mathrm{M}_{\mathrm{B}} \mathrm{M}_{\mathrm{D}}, & \operatorname{Pr}_{\mathrm{M}_{\mathrm{B}} \mathrm{M}_{\mathrm{D}}=\frac{\theta_{\mathrm{B}} \theta_{\mathrm{D}}}{4 \pi^{2}},} \\ \mathrm{M}_{\mathrm{B}} \mathrm{m}_{\mathrm{D}}, & \operatorname{Pr}_{\mathrm{M}_{\mathrm{B}} \mathrm{m}_{\mathrm{D}}=\frac{\theta_{\mathrm{B}}\left(2 \pi-\theta_{\mathrm{D}}\right)}{4 \pi^{2}},} \\ \mathrm{~m}_{\mathrm{B}} \mathrm{M}_{\mathrm{D}}, & \operatorname{Pr}_{\mathrm{m}_{\mathrm{B}} \mathrm{M}_{\mathrm{D}}=\frac{\left(2 \pi-\theta_{\mathrm{B}}\right) \theta_{\mathrm{D}}}{4 \pi^{2}},} \\ \mathrm{~m}_{\mathrm{B}} \mathrm{m}_{\mathrm{D}}, & \operatorname{Pr}_{\mathrm{m}_{\mathrm{B}} \mathrm{m}_{\mathrm{D}}}=\frac{\left(2 \pi-\theta_{\mathrm{B}}\right)\left(2 \pi-\theta_{\mathrm{D}}\right)}{4 \pi^{2}},\end{cases}
$$

where $M_{B}, m_{B}$, and $\theta_{B}$ are the main lobe gain, side lobe gain, and half power beamwidth of the mmWave BS antenna, and $\mathrm{M}_{\mathrm{D}}, \mathrm{m}_{\mathrm{D}}$, and $\theta_{\mathrm{D}}$ are the main lobe gain, side lobe gain, and half power beamwidth of the mmMD antenna.

In light of the blockage effects in the outdoor scenario, a mmMD is associated with either a LoS mmWave BS or a NLoS mmWave BS. We denote $f_{\operatorname{Pr}}(R)$ as the probability that a link at a distance $R$ is LoS, while the NLoS probability of a link is $1-f_{\operatorname{Pr}}(R)$. We consider two different path loss laws: $L(R)=\beta_{\mathrm{LoS}}^{\mathrm{mm}} R^{-\alpha_{\mathrm{LoS}}^{\mathrm{mm}}}$ is the pathloss function for LoS channel and $L(R)=\beta_{\mathrm{NLoS}}^{\mathrm{mm}} R^{-\alpha_{\mathrm{NLOS}}^{\mathrm{mm}}}$ is the pathloss function for NLoS channel, where $\beta_{\mathrm{LoS}}^{\mathrm{mm}}, \beta_{\mathrm{NLoS}}^{\mathrm{mm}}$ are the frequency dependent constant values and $\alpha_{\mathrm{LoS}}^{\mathrm{mm}}, \alpha_{\mathrm{NLoS}}^{\mathrm{mm}}$ are the pathloss exponents.

For a typical mmMD $o$ connected with its serving mmWave $\mathrm{BS}$, its instantaneous harvested power is written as

$$
\begin{aligned}
& P_{r}^{\mathrm{mm}}= \underbrace{\eta_{\mathrm{mm}} P_{\mathrm{mm}} \mathrm{M}_{\mathrm{B}} \mathrm{M}_{\mathrm{D}} L\left(\left|Y_{o}\right|\right)}_{\Lambda_{\mathrm{mm}_{1}}} \\
&+\underbrace{\eta_{\mathrm{mm}} \sum_{\ell \in \Phi_{\mathrm{mm}}\left(\lambda_{\mathrm{mm}}\right) \backslash\{o\}} P_{\mathrm{mm}} G_{\ell} L\left(\left|Y_{\ell, \mathrm{mm}}\right|\right)}_{\Lambda_{\mathrm{mm}_{2}}},
\end{aligned}
$$

where $\Lambda_{\mathrm{mm}_{1}}$ is the directed transferred power, and $\Lambda_{\mathrm{mm}_{2}}$ is the total power from the ambient mmWave $\mathrm{RF}, \eta_{\mathrm{mm}}$ is the mmWave RF-to-DC conversion efficiency, $P_{\mathrm{mm}}$ is the mmWave BS transmit power, $\left|Y_{o}\right|$ is the distance between the typical mmMD and its serving mmWave $\mathrm{BS}$, and $\left|Y_{\ell, \mathrm{mm}}\right|$ is the distance between the typical $\mathrm{mmMD}$ and the mmWave BS $\ell \in \Phi_{\mathrm{mm}} \backslash\{o\}$ (except the serving mmWave BS).

\section{ENERGY HARVESTING EVALUATION}

We evaluate the wireless energy harvesting in the sub-6 $\mathrm{GHz}$ and mmWave cellular networks. To comprehensively understand it, we respectively examine the directed transferred power and the ambient RF harvested power that a mobile device can obtain.

\section{A. Directed Transferred Power}

1) Sub-6 GHz Tier: In sub-6 GHz tier, given a power threshold $P_{\text {th }}$, the coverage probability that the directed transferred power is larger than $P_{\text {th }}$ can be derived as

$$
\begin{aligned}
\bar{\Psi}_{\mathrm{D}}^{\mu}\left(P_{\mathrm{th}}\right)= & \sum_{n=0}^{N-1}\left(\frac{P_{\mathrm{th}}}{\eta_{\mu} P_{\mu} \beta_{\mu}}\right)^{n} \frac{2 \pi \lambda_{\mu}}{n !} \times \\
& \int_{0}^{\infty} e^{-\frac{r^{\alpha}{ }_{P_{\mathrm{th}}}}{\eta_{\mu} P_{\mu} \beta_{\mu}}-\pi \lambda_{\mu} r^{2}} r^{\alpha_{\mu} n+1} d r .
\end{aligned}
$$

Proof: Based on (1), $\bar{\Psi}_{\mathrm{D}}^{\mu}\left(P_{\mathrm{th}}\right)$ is calculated as

$$
\begin{aligned}
& \bar{\Psi}_{\mathrm{D}}^{\mu}\left(P_{\mathrm{th}}\right)=\operatorname{Pr}\left(\Lambda_{\mu_{1}}>P_{\mathrm{th}}\right) \\
& =\int_{0}^{\infty} \operatorname{Pr}\left(\eta_{\mu} P_{\mu}\left\|\mathbf{h}_{o}\right\|^{2} \beta_{\mu} r^{-\alpha_{\mu}}>P_{\mathrm{th}}\right) f_{\left|X_{o}\right|}(r) d r,
\end{aligned}
$$

where $f_{\left|X_{o}\right|}(r)$ is the probability density function (PDF) of the distance between a mobile device and its nearest BS, which is given by

$$
f_{\left|X_{o}\right|}(r)=2 \pi \lambda_{\mu} r \exp \left(-\pi \lambda_{\mu} r^{2}\right) .
$$

Considering that $\left\|\mathbf{h}_{o}\right\|^{2} \sim \Gamma(N, 1)$, we can further derive (5) as

$$
\bar{\Psi}_{\mathrm{D}}^{\mu}\left(P_{\mathrm{th}}\right)=\sum_{n=0}^{N-1} \int_{0}^{\infty} \frac{e^{-\frac{r^{\alpha} \mu P_{\mathrm{th}}}{\eta_{\mu} P_{\mu} \beta}}}{n !}\left(\frac{r^{\alpha} P_{\mathrm{th}}}{\eta_{\mu} P_{\mu} \beta_{\mu}}\right)^{n} f_{\left|X_{o}\right|}(r) d r .
$$

Substituting (6) into (7), we obtain (4).

Based on (4), there is one sufficient condition for $\bar{\Psi}_{\mathrm{D}}^{\mu}\left(P_{\mathrm{th}}\right)>\varepsilon(0<\varepsilon<1)$ given a specific $P_{\mathrm{th}}$, which is shown as follows.

Corollary 1. The probability of the achievable directed transferred power $P_{\mathrm{th}}$ can be larger than $\varepsilon$, if the sub-6 GHzBS density satisfies

$$
\lambda_{\mu}>\left(\frac{\eta_{\mu} P_{\mu} \beta_{\mu} N}{P_{\mathrm{th}}}\right)^{-2 / \alpha_{\mu}} \frac{\ln \frac{1}{1-\varepsilon}}{\pi}
$$

with large $N$. 
Proof: With large $N,\left\|\mathbf{h}_{o}\right\|^{2} \approx N$ [9], (5) can be approximated as

$$
\begin{aligned}
\widetilde{\Psi}_{\mathrm{D}}^{\mu}\left(P_{\mathrm{th}}\right) & =\int_{0}^{\left(\frac{\eta_{\mu} P_{\mu} \beta_{\mu} N}{P_{\mathrm{th}}}\right)^{1 / \alpha_{\mu}}} f_{\left|X_{o}\right|}(r) d r \\
& =1-\exp \left(-\pi \lambda_{\mu}\left(\frac{\eta_{\mu} P_{\mu} \beta_{\mu} N}{P_{\mathrm{th}}}\right)^{2 / \alpha_{\mu}}\right) .
\end{aligned}
$$

Let $\widetilde{\Psi}_{\mathrm{D}}^{\mu}\left(P_{\mathrm{th}}\right)>\varepsilon$, after some manipulations, we get (8).

It is indicated from Corollary 1 that the number of sub-6 $\mathrm{GHz}$ BSs needs to be large enough for WPT.

2) MmWave Tier: In mmWave tier, the coverage probability that the directed transferred power is larger than a threshold $P_{\text {th }}$ can be derived as

$$
\begin{gathered}
\bar{\Psi}_{\mathrm{D}}^{\mathrm{mm}}\left(P_{\mathrm{th}}\right)=2 \pi \lambda_{\mathrm{mm}}[ \\
\int_{0}^{\varsigma\left(\beta_{\mathrm{LoS}}^{\mathrm{mm}}, \alpha_{\mathrm{LoS}}^{\mathrm{mm}}\right)} y f_{\mathrm{Pr}}(y) e^{-2 \pi \lambda_{\mathrm{mm}}\left[\Theta(y)+\Xi\left(\varphi_{\mathrm{LoS}}(y)\right)\right]} d y+ \\
\left.\int_{0}^{\varsigma\left(\beta_{\mathrm{NLOS}}^{\mathrm{mm}}, \alpha_{\mathrm{NLoS}}^{\mathrm{mm}}\right)} y\left(1-f_{\operatorname{Pr}}(y)\right) e^{-2 \pi \lambda_{\mathrm{mm}}\left[\Theta\left(\varphi_{\mathrm{NLoS}}(y)\right)+\Xi(y)\right]} d y\right],
\end{gathered}
$$

$$
\text { where } \varsigma\left(y_{1}, y_{2}\right)=\left(\frac{\eta_{\mathrm{mm}} P_{\mathrm{mm}} \mathrm{M}_{\mathrm{B}} \mathrm{M}_{\mathrm{D}}}{P_{\mathrm{th}}} y_{1}\right)^{1 / y_{2}} \text {, }
$$
$\Theta(y)=\int_{0}^{y} t f_{\operatorname{Pr}}(t) d t, \Xi(y) \underset{1 / \mathrm{mm}^{2}}{=} \int_{0}^{y}\left(1-f_{\operatorname{Pr}}(t)\right) t d t$, $\varphi_{\mathrm{LoS}}(y)=\left(\frac{\beta_{\mathrm{NLoS}}^{\mathrm{mm}}}{\beta_{\mathrm{LoS}}^{\mathrm{mos}}}\right)^{1 / \alpha_{\mathrm{NLOS}}^{\mathrm{mm}}} y^{\alpha_{\mathrm{LoS}}^{\mathrm{mm}} / \alpha_{\mathrm{NLOS}}^{\mathrm{mm}}}, \quad$ and $\varphi_{\mathrm{NLoS}}(y)=\left(\frac{\beta_{\mathrm{LoS}}^{\mathrm{mm}}}{\beta_{\mathrm{NLoS}}^{\mathrm{moS}}}\right)^{1 / \alpha_{\mathrm{LoS}}^{\mathrm{mm}}} y^{\alpha_{\mathrm{NLoS}}^{\mathrm{mm}} / \alpha_{\mathrm{LoS}}^{\mathrm{mm}}}$.

Proof: Based on (3), $\bar{\Psi}_{\mathrm{D}}^{\mathrm{mm}}\left(P_{\mathrm{th}}\right)$ is calculated as

$$
\begin{aligned}
\bar{\Psi}_{\mathrm{D}}^{\mathrm{mm}}\left(P_{\mathrm{th}}\right) & =\operatorname{Pr}\left(\Lambda_{\mathrm{mm}_{1}}>P_{\mathrm{th}}\right) \\
& =\Upsilon_{\mathrm{LoS}} \int_{0}^{\varsigma\left(\beta_{\mathrm{LoS}}^{\mathrm{mm}}, \alpha_{\mathrm{LoS}}^{\mathrm{mm}}\right)} f_{R}^{\mathrm{LoS}}(y) d y+ \\
& \Upsilon_{\mathrm{NLoS}} \int_{0}^{\varsigma\left(\beta_{\mathrm{NLoS}}^{\mathrm{mm}}, \alpha_{\mathrm{NLoS}}^{\mathrm{mm}}\right)} f_{R}^{\mathrm{NLoS}}(y) d y,
\end{aligned}
$$

where $\varsigma\left(x_{1}, x_{2}\right)=\left(\frac{\eta_{\mathrm{mm}} P_{\mathrm{mm}} \mathrm{M}_{\mathrm{B}} \mathrm{M}_{\mathrm{D}}}{P_{\mathrm{th}}} x_{1}\right)^{1 / x_{2}}, \Upsilon_{\mathrm{LoS}}$ represents the probability that the typical user is connected to a LoS BS and $\Upsilon_{\mathrm{NLOS}}=1-\Upsilon_{\mathrm{LoS}}$ represents the probability that the typical user is connected to a NLoS BS, $f_{R}^{\operatorname{LoS}}(x)$ is the conditioned PDF of the distance between the typical mmMD and its serving LoS BS, and $f_{R}^{\mathrm{NLoS}}(x)$ is the conditioned PDF of the distance between the typical mmMD and its serving NLoS BS, which are given by [10]

$$
f_{R}^{\mathrm{LoS}}(y)=\frac{2 \pi \lambda_{\mathrm{mm}}}{\Upsilon_{\mathrm{LoS}}} y f_{\mathrm{Pr}}(y) e^{-2 \pi \lambda_{\mathrm{mm}}\left[\Theta(y)+\Xi\left(\varphi_{\mathrm{LoS}}(y)\right)\right]},
$$

and

$f_{R}^{\mathrm{NLoS}}(y)=\frac{2 \pi \lambda_{\mathrm{mm}}}{\Upsilon_{\mathrm{NLoS}}} y\left(1-f_{\mathrm{Pr}}(y)\right) e^{-2 \pi \lambda_{\mathrm{mm}}\left[\Theta\left(\varphi_{\mathrm{NLoS}}(y)\right)+\Xi(y)\right]}$,

respectively.

According to Corollary 1 and (10), we have the following important insight.
Corollary 2. Given a threshold $P_{\mathrm{th}}$, the coverage probability of the directed power transfer in the mmWave cell is larger than that in the sub-6 GHz cell under the following condition:

$$
\lambda_{\mu}<\frac{\ln \left(1-\bar{\Psi}_{\mathrm{D}}^{\mathrm{mm}}\left(P_{\mathrm{th}}\right)\right)^{-1}}{\pi\left(\frac{\eta_{\mu} P_{\mu} \beta N}{P_{\mathrm{th}}}\right)^{2 / \alpha_{\mu}}}
$$

with large $N$. In particular, when only LoS mmWave links are utilized for power transfer, the above condition is transformed as

$$
\frac{\lambda_{\mu}}{\lambda_{\mathrm{mm}}}<\frac{\xi^{2}}{\left(\frac{\eta_{\mu} P_{\mu} \beta N}{P_{\mathrm{th}}}\right)^{2 / \alpha_{\mu}}},
$$

where $\xi=\min \left\{R_{\mathrm{LoS}}, \varsigma\left(\beta_{\mathrm{LoS}}^{\mathrm{mm}}, \alpha_{\mathrm{LoS}}^{\mathrm{mm}}\right)\right\}$, and $R_{\mathrm{LoS}}$ denotes the maximum distance that LoS can be guaranteed [10, 11].

Proof: Based on the LoS mmWave model in [10,11], the probability that the directed transferred power is larger than a threshold $P_{\text {th }}$ under LoS mmWave power transfer is calculated as

$$
\begin{aligned}
& \bar{\Psi}_{\mathrm{D}}^{\mathrm{mm}}\left(P_{\mathrm{th}}\right) \\
& =\operatorname{Pr}\left(\eta_{\mathrm{mm}} P_{\mathrm{mm}} \mathrm{M}_{\mathrm{B}} \mathrm{M}_{\mathrm{D}} L\left(\left|Y_{o}\right|\right) \Phi\left(\left|Y_{o}\right|<R_{\mathrm{LoS}}\right)>P_{\mathrm{th}}\right) \\
& \stackrel{(\mathrm{a})}{=} \int_{0}^{\xi} 2 \pi \lambda_{\mathrm{mm}} r \exp \left(-\pi \lambda_{\mathrm{mm}} r^{2}\right) d r \\
& =1-\exp \left(-\pi \lambda_{\mathrm{mm}} \xi^{2}\right)
\end{aligned}
$$

where $\Phi(\cdot)$ is the indicator function that returns one if the condition is satisfied and zero otherwise, $\xi=$ $\min \left\{R_{\mathrm{LoS}}, \varsigma\left(\beta_{\mathrm{LoS}}^{\mathrm{mm}}, \alpha_{\mathrm{LoS}}^{\mathrm{mm}}\right)\right\}$, step (a) is obtained by considering the fact that devices try to be connected to the nearest BSs such that LoS may exist. Substituting (16) into (14), we obtain (15) and complete the proof.

It is indicated from Corollary 2 that mmWave WPT can be better than sub-6 GHz WPT when the number of sub- $6 \mathrm{GHz}$ BSs is not sufficiently deployed, compared to the number of mmWave BSs.

\section{B. Ambient RF Harvested Power}

In order to avoid singularity at zero distance and ensure the finite moments of the sum of the ambient RF signals, we incorporate the distance constraint into the path loss function, which is $\tilde{L}(|X|)=\beta\left(\max \left(r_{o},|X|\right)\right)^{-\alpha}$ with a reference distance $r_{o} \geq 1[12,13]$. It should be noted that the reference distance can also represent the minimum distance between a BS and the typical UE in the practical scenario, and has no effect on the evaluation of the energy coverage probability, since the harvested energy is usually larger than the predefined threshold when $|X| \leq r_{o}$.

1) Sub-6 GHz Tier: In sub-6 GHz tier, let $\bar{\Psi}_{\mathrm{A}}^{m m}\left(P_{\mathrm{th}}\right)$ denote the probability that the ambient RF harvested power is larger than a threshold $P_{\mathrm{th}}, \bar{\Psi}_{\mathrm{A}}^{\mu}\left(P_{\mathrm{th}}\right)$ is upper bounded as

$$
\bar{\Psi}_{\mathrm{A}}^{\mu}\left(P_{\mathrm{th}}\right) \leq \min \left\{\operatorname{var}[\Xi] /\left(\frac{P_{\mathrm{th}}}{\eta_{\mu} P_{\mu}}-\mathbb{E}[\Xi]\right)^{2}, 1\right\},
$$

where $\mathbb{E}[\Xi]$ and $\operatorname{var}[\Xi]$ are respectively given by (18) and (19) at the top of next page. 


$$
\mathbb{E}[\Xi]=\beta_{\mu} 2 \pi \lambda_{\mu} \int_{0}^{\infty}\left(\max \left(r_{o}, t\right)\right)^{-\alpha_{\mu}} t\left(1-\exp \left(-\pi \lambda_{\mu} t^{2}\right)\right) d t
$$

$$
\operatorname{var}[\Xi]=\beta_{\mu}^{2} 4 \pi \lambda_{\mu} \int_{0}^{\infty}\left(\max \left(r_{o}, t\right)\right)^{-2 \alpha_{\mu}} t\left(1-\exp \left(-\pi \lambda_{\mu} t^{2}\right)\right) d t
$$

Proof: Based on (1), $\bar{\Psi}_{\mathrm{A}}^{\mu}\left(P_{\mathrm{th}}\right)$ is calculated as

$$
\begin{aligned}
& \bar{\Psi}_{\mathrm{A}}^{\mu}\left(P_{\mathrm{th}}\right)=\operatorname{Pr}\left(\Lambda_{\mu_{2}}>P_{\mathrm{th}}\right) \\
& =\operatorname{Pr}(\underbrace{\left.\sum_{k \in \Phi_{\mu}\left(\lambda_{\mu}\right) \backslash\{o\}}\left|\mathbf{h}_{k, o} \frac{\mathbf{h}_{k}^{\dagger}}{\left\|\mathbf{h}_{k}\right\|}\right|^{2} \tilde{L}\left(\left|X_{k, \mu}\right|\right)>\frac{P_{\mathrm{th}}}{\eta_{\mu} P_{\mu}}\right)}_{\Xi} \\
& \stackrel{(b)}{\leq} \min \left\{\frac{\operatorname{var}[\Xi]}{\left(\frac{P_{\mathrm{th}}}{\eta_{\mu} P_{\mu}}-\mathbb{E}[\Xi]\right)^{2}}, 1\right\},
\end{aligned}
$$

where step (b) is obtained by using the Chebyshev's inequality. Here, $\mathbb{E}[\Xi]$ and var $[\Xi]$ denote the expectation and variance of $\Xi$, respectively.

We first derive $\mathbb{E}[\Xi]$. By using the Campbell's theorem [14], $\mathbb{E}[\Xi]$ is given by

$$
\begin{aligned}
& \mathbb{E}[\Xi]=\mathbb{E}\left[\left|\mathbf{h}_{k, o} \frac{\mathbf{h}_{k}^{\dagger}}{\left\|\mathbf{h}_{k}\right\|}\right|^{2}\right] \beta_{\mu} \\
& \times 2 \pi \lambda_{\mu} \int_{0}^{\infty} \int_{r}^{\infty}\left(\max \left(r_{o}, t\right)\right)^{-\alpha_{\mu}} t f_{\left|X_{o}\right|}(r) d t d r,
\end{aligned}
$$

where $f_{\left|X_{o}\right|}(r)$ is given by (6). Based on [9, Proposition 1], we note that $\left|\mathbf{h}_{k, o} \frac{\mathbf{h}_{k}^{\dagger}}{\left\|\mathbf{h}_{k}\right\|}\right|^{2} \sim \exp (1)$, thus $\mathbb{E}\left[\left|\mathbf{h}_{k, o} \frac{\mathbf{h}_{k}^{\dagger}}{\left\|\mathbf{h}_{k}\right\|}\right|^{2}\right]=1$. Changing the order of integration in (21), we obtain (18).

The variance of $\Xi$ is derived as

$$
\begin{aligned}
& \operatorname{var}[\Xi]=\left.\frac{\partial^{2}}{\partial s^{2}} \mathbb{E}[\exp (s \Xi)]\right|_{s=0}-(\mathbb{E}[\Xi])^{2} \\
& \left.\stackrel{(c)}{=} \mathbb{E}\left[\left|\mathbf{h}_{k, o} \frac{\mathbf{h}_{k}^{\dagger}}{\left\|\mathbf{h}_{k}\right\|}\right|^{4}\right] \beta_{\mu}^{2}\right] \\
& \times 2 \pi \lambda_{\mu} \int_{0}^{\infty} \int_{r}^{\infty}\left(\max \left(r_{o}, t\right)\right)^{-2 \alpha_{\mu}} t f_{\left|X_{o}\right|}(r) d t d r
\end{aligned}
$$

where step (c) is obtained by using the modified Campbell's theorem. Since $\mathbb{E}\left[\left|\mathbf{h}_{k, o} \frac{\mathbf{h}_{k}^{\dagger}}{\left\|\mathbf{h}_{k}\right\|}\right|^{4}\right]=2$, we can finally obtain (19) and complete the proof.

2) MmWave Tier: In mmWave tier, let $\bar{\Psi}_{\mathrm{A}}^{\mathrm{mm}}\left(P_{\mathrm{th}}\right)$ denote the probability that the ambient RF harvested power is larger than a threshold $P_{\mathrm{th}}$, given $\varepsilon, \bar{\Psi}_{\mathrm{A}}^{\mathrm{mm}}\left(P_{\mathrm{th}}\right)>\varepsilon$ holds when the mmWave BS density meets

$$
\lambda_{\mathrm{mm}}>\left(\frac{P_{\mathrm{th}}}{\vartheta_{\mathrm{mm}} \eta_{\mathrm{mm}} P_{\mathrm{mm}}}\right)^{\frac{2}{\alpha_{\mathrm{LoS}}^{\ln }}}
$$

where $\vartheta_{\mathrm{mm}}$ is a constant value defined by

$$
\operatorname{Pr}\left(\sum_{\ell \in \Phi_{\mathrm{mm}}(1) \backslash\{o\}} G_{\ell} \Delta\left(\left|Y_{\ell, \mathrm{mm}}\right|\right)>\vartheta_{\mathrm{mm}}\right)=\varepsilon
$$

with $\Delta\left(\left|Y_{\ell, \mathrm{mm}}\right|\right)=\tilde{L}_{\mathrm{LoS}}\left(\left|Y_{\ell, \mathrm{mm}}\right|\right) \amalg\left(f_{\mathrm{Pr}}\left(\left|Y_{\ell, \mathrm{mm}}\right|\right)\right)$, here, $\amalg(x)$ denotes Bernoulli distribution.

Proof: Based on (3), the probability that the ambient RF harvested power $\Lambda_{\mathrm{mm}_{2}}$ is larger than a threshold $P_{\mathrm{th}}$ can be obtained as

$$
\begin{aligned}
& \bar{\Psi}_{\mathrm{A}}^{\mathrm{mm}}\left(P_{\mathrm{th}}\right)=\operatorname{Pr}\left(\Lambda_{\mathrm{mm}_{2}}>P_{\mathrm{th}}\right) \\
= & \operatorname{Pr}\left(\sum_{\ell \in \Phi_{\mathrm{mm}}\left(\lambda_{\mathrm{mm}}\right) \backslash\{o\}} G_{\ell} \tilde{L}\left(\left|Y_{\ell, \mathrm{mm}}\right|\right)>\frac{P_{\mathrm{th}}}{\eta_{\mathrm{mm}} P_{\mathrm{mm}}}\right) .
\end{aligned}
$$

Since the ambient RF energy from the NLoS BSs is negligible, $\bar{\Psi}_{\mathrm{A}}^{\mathrm{mm}}\left(P_{\mathrm{th}}\right)$ can be lower-bounded as

$$
\begin{aligned}
& \bar{\Psi}_{\mathrm{A}}^{\mathrm{mm}}\left(P_{\mathrm{th}}\right) \\
& \geq \operatorname{Pr}\left(\sum_{\ell \in \Phi_{\mathrm{mm}}\left(\lambda_{\mathrm{mm}}\right) \backslash\{o\}} G_{\ell} \Delta\left(\left|Y_{\ell, \mathrm{mm}}\right|\right)>\frac{P_{\mathrm{th}}}{\eta_{\mathrm{mm}} P_{\mathrm{mm}}}\right) \\
& \stackrel{(d)}{=} \operatorname{Pr}\left(\sum_{\ell \in \Phi_{\mathrm{mm}}(1) \backslash\{o\}} G_{\ell} \Delta\left(\left|Y_{\ell, \mathrm{mm}}\right|\right)>\lambda_{\mathrm{mm}}^{-\frac{\alpha_{\mathrm{LoS}}^{\mathrm{mm}}}{2}} \frac{P_{\mathrm{th}}}{\eta_{\mathrm{mm}} P_{\mathrm{mm}}}\right),
\end{aligned}
$$

where step (d) is obtained by Mapping theorem [14].

Given $\varepsilon$, we define the constant $\vartheta_{\mu}$ as (24). Then, we can directly obtain $\bar{\Psi}_{\mathrm{A}}^{\mu}\left(P_{\mathrm{th}}\right)>\varepsilon$ if and only if condition (23) is satisfied, and complete the proof.

Based on (17) and (23), we have the following corollary:

Corollary 3. The ambient $R F$ energy harvesting in the mmWave cell outperforms that in the sub-6 GHz cell under the condition (23), where $\vartheta_{\mathrm{mm}}$ is given by (24) with $\varepsilon=$ $\min \left\{\operatorname{var}[\Xi] /\left(\frac{P_{\mathrm{th}}}{\eta_{\mu} P_{\mu}}-\mathbb{E}[\Xi]\right)^{2}, 1\right\}$.

\section{Power Transfer Mode Selection}

In the above, we have illustrated the wireless energy harvesting in sub- $6 \mathrm{GHz}$ and mmWave tiers. In this subsection, we consider mode selection for WPT in hybrid cellular networks, i.e., mobile devices have the ability of selecting sub-6 $\mathrm{GHz}$ or mmWave tier for maximizing the directed transferred power, since the amount of harvested energy from ambient $\mathrm{RF}$ is much smaller compared to that from directed power transfer $[1,4,5]$. Thus, we have the following Propositions:

Proposition 1. The probability that a mobile device selects the sub-6 GHz WPT is given by

$$
\begin{aligned}
\mathcal{H}_{\mu} & =2 \pi \lambda_{\mu} \int_{0}^{\infty} r \exp \left(-2 \pi \lambda_{\operatorname{mm}}\left(\int_{0}^{\hat{R}_{\mathrm{LoS}}^{\mathrm{mm}}(r)} f_{\mathrm{Pr}}(t) t d t\right.\right. \\
& \left.\left.+\int_{0}^{\hat{R}_{\mathrm{NLoS}}^{\mathrm{mm}}(r)}\left(1-f_{\operatorname{Pr}}(t)\right) t d t\right)-\pi \lambda_{\mu} r^{2}\right) d r
\end{aligned}
$$


where $\hat{R}_{\mathrm{LoS}}^{\mathrm{mm}}(r)=\left(\varpi \frac{\beta_{\mathrm{LoS}}^{\mathrm{mm}}}{\beta_{\mu}}\right)^{1 / \alpha_{\mathrm{LoS}}^{\mathrm{mm}}} r^{\frac{\alpha_{\mu}}{\alpha_{\mathrm{LoS}}^{\mathrm{mm}}}}$ with $\varpi=$ $\frac{\eta_{\mathrm{mm}} P_{\mathrm{mmm}} \mathrm{M}_{\mathrm{B}} \mathrm{M}_{\mathrm{D}}}{\eta_{\mu} P_{\mu} N}, \hat{R}_{\mathrm{NLoS}}^{\mathrm{mm}}(r)=\left(\varpi \frac{\beta_{\mathrm{NLoS}}^{\mathrm{mm}}}{\beta_{\mu}}\right)^{1 / \alpha_{\mathrm{NLoS}}^{\mathrm{mm}}} r^{\frac{\alpha_{\mu}}{\alpha_{\mathrm{NLoS}}^{\mathrm{m} m}}}$.

Proof: We note that in the mmWave cell, the smallscale fading is negligible, and the directed transferred power is dominated by the mmWave pathloss. In the sub- $6 \mathrm{GHz}$ cell, the small-scale fading is averaged out when the BS antennas are large, i.e., $\left\|\mathbf{h}_{o}\right\|^{2} \approx N$. Therefore, the probability that a mobile device selects the sub-6 GHz WPT can be expressed as

$$
\begin{aligned}
& \mathcal{H}_{\mu}=\operatorname{Pr}\left(\Lambda_{\mu_{1}}>\Lambda_{\mathrm{mm}_{1}}\right) \\
& =\mathbb{E}_{\left|X_{o}\right|}\left[\operatorname{Pr}\left(\eta_{\mu} P_{\mu} N L\left(\left|X_{o}\right|\right)>\eta_{\mathrm{mm}} P_{\mathrm{mm}} \mathrm{M}_{\mathrm{B}} \mathrm{M}_{\mathrm{D}} L(R)\right)\right] \\
& \stackrel{(e)}{=} \mathbb{E}_{\left|X_{o}\right|}[\underbrace{\operatorname{Pr}\left(R_{\ell}^{\mathrm{mm}}>\left(\varpi \frac{\beta_{\mathrm{LoS}}^{\mathrm{mm}}}{\beta_{\mu}}\right)^{1 / \alpha_{\mathrm{LoS}}^{\mathrm{mm}}}\left|X_{o}\right|^{\frac{\alpha_{\mu}}{\alpha_{\mathrm{LoS}}^{\mathrm{mmm}}}} \mid \ell \in \Phi_{\mathrm{mm}}^{\mathrm{LoS}}\right)}_{\Theta_{\mathrm{L}}\left(\left|X_{o}\right|\right)} \\
& \times \underbrace{\operatorname{Pr}\left(R_{\ell}^{\mathrm{mm}}>\left(\varpi \frac{\beta_{\mathrm{NLoS}}^{\mathrm{mm}}}{\beta_{\mu}}\right)^{1 / \alpha_{\mathrm{NLOS}}^{\mathrm{mm}}}\left|X_{o}\right|^{\left.\frac{\alpha_{\mu}}{\alpha_{\mathrm{NLOS}}^{\mathrm{mLn}}} \mid \ell \in \Phi_{\mathrm{mm}}^{\mathrm{NLoS}}\right)}\right]}_{\Theta_{\mathrm{N}}\left(\left|X_{o}\right|\right)} \\
& =\int_{0}^{\infty} \Theta_{\mathrm{L}}(r) \Theta_{\mathrm{N}}(r) f_{\left|X_{o}\right|}(r) d r,
\end{aligned}
$$

where step (e) is obtained by considering two independent LoS BS process $\Phi_{\mathrm{mm}}^{\mathrm{LoS}}$ and NLoS BS process $\Phi_{\mathrm{mm}}^{\mathrm{NLoS}}, \varpi=$ $\frac{\eta_{\mathrm{mm}} P_{\mathrm{mm}} \mathrm{M}_{\mathrm{B}} \mathrm{M}_{\mathrm{D}}}{\eta_{\mu} P_{\mu} N}, f_{\left|X_{o}\right|}(r)$ is the PDF of $\left|X_{o}\right|$ given in (6). By employing the void probability, we can obtain $\Theta_{\mathrm{L}}$ as

$$
\begin{aligned}
\Theta_{\mathrm{L}}(r)=\operatorname{Pr}( & \text { No LoS mmWave BS } \\
& \text { closer than } \left.\hat{R}_{\mathrm{LoS}}^{\mathrm{mm}}(r)\right) \\
= & \exp \left(-2 \pi \lambda_{\mathrm{mm}} \int_{0}^{\hat{R}_{\mathrm{LoS}}^{\mathrm{mm}}(r)} f_{\mathrm{Pr}}(t) t d t\right),
\end{aligned}
$$

where $\hat{R}_{\mathrm{LoS}}^{\mathrm{mm}}(r)=\left(\varpi \frac{\beta_{\mathrm{LoS}}^{\mathrm{mm}}}{\beta_{\mu}}\right)^{1 / \alpha_{\mathrm{LoS}}^{\mathrm{mm}}} r^{\frac{\alpha_{\mu}}{\alpha_{\mathrm{LoS}}^{\mathrm{mm}}}}$. Similar to (29), $\Theta_{\mathrm{N}}$ is given by

$\Theta_{\mathrm{N}}(r)=\exp \left(-2 \pi \lambda_{\mathrm{mm}} \int_{0}^{\hat{R}_{\mathrm{NLoS}}^{\mathrm{mm}}(r)}\left(1-f_{\mathrm{Pr}}(t)\right) t d t\right)$,

where $\hat{R}_{\mathrm{NLoS}}^{\mathrm{mm}}(r)=\left(\varpi \frac{\beta_{\mathrm{NLoS}}^{\mathrm{mm}}}{\beta_{\mu}}\right)^{1 / \alpha_{\mathrm{NLoS}}^{\mathrm{mm}}} r^{\frac{\alpha_{\mu}}{\alpha_{\mathrm{NLOS}}^{\mathrm{m} m}}}$. Substituting (29) and (30) into (28), we obtain (27).

Proposition 2. The probability that a mobile device selects a LoS mmWave BS for the mmWave WPT is given by

$$
\begin{aligned}
\mathcal{H}_{\mathrm{mm}}^{\mathrm{LoS}} & =2 \pi \lambda_{\mathrm{mm}} \int_{0}^{\infty} r f_{\mathrm{Pr}}(r) \exp \left(-2 \pi \lambda_{\mathrm{mm}}\left(\int_{0}^{r} f_{\mathrm{Pr}}(t) t d t\right.\right. \\
& \left.\left.+\int_{0}^{\widetilde{R}_{\mathrm{NLoS}}^{\mathrm{mm}}(r)}\left(1-f_{\mathrm{Pr}}(t)\right) t d t\right)-\lambda_{\mu} \widetilde{A}_{\mu}(r)\right) d r, \quad(31)
\end{aligned}
$$

where $\widetilde{R}_{\mathrm{NLOS}}^{\operatorname{mm}}(r)=\left(\frac{\beta_{\mathrm{NLoS}}^{\operatorname{mm}}}{\beta_{\mathrm{LoS}}^{\operatorname{mm}}}\right)^{1 / \alpha_{\mathrm{NLoS}}^{m m}} r^{\frac{\alpha_{\mathrm{LoS}}^{\operatorname{mm}}}{\alpha_{\mathrm{NLOS}}^{\mathrm{mLO}}}}$ and $\widetilde{A}_{\mu}(r)=$ $\pi\left(\frac{\beta_{\mu}}{\varpi \beta_{\mathrm{LoS}}^{m m}}\right)^{2 / \alpha_{\mu}} r^{\frac{2 \alpha_{\mathrm{LoS}}^{\mathrm{mm}}}{\alpha_{\mu}}}$. Then, the probability that a mobile device selects a NLoS mmWave BS for the mmWave WPT is $\mathcal{H}_{\mathrm{mm}}^{\mathrm{NLoS}}=1-\mathcal{H}_{\mu}-\mathcal{H}_{\mathrm{mm}}^{\mathrm{LoS}}$.
TABLE I

SimUlATION PARAMETERS

\begin{tabular}{|l|c|}
\hline Parameter & Value \\
\hline sub-6 GHz carrier frequency & $f_{c}=1.5 \mathrm{GHz}$ \\
\hline MmWave carrier frequency & $f_{\mathrm{mm}}=28 \mathrm{GHz}$ \\
\hline RF-to-DC conversion efficiency & $\eta_{\mu}=\eta_{\mathrm{mm}}=0.6$ \\
\hline BS transmit power & $P_{\mu}=P_{\mathrm{mm}}=30 \mathrm{dBm}$ \\
\hline Reference distance & $r_{o}=1$ \\
\hline MmWave pathloss exponent & $\alpha_{\mathrm{LoS}}^{\mathrm{mm}}=2, \alpha_{\mathrm{NLoS}}^{\mathrm{mm}}=2.9$ \\
\hline \multirow{2}{*}{ MmWave antenna beam pattern } & $\begin{array}{c}\mathrm{BS}:\left(\mathrm{M}_{\mathrm{B}}, \mathrm{m}_{\mathrm{B}}, \theta_{\mathrm{B}}\right)=\left(18 \mathrm{~dB},-2 \mathrm{~dB}, 10^{\circ}\right) \\
\text { MD: }\left(\mathrm{M}_{\mathrm{D}}, \mathrm{m}_{\mathrm{D}}, \theta_{\mathrm{D}}\right)=\left(10 \mathrm{~dB},-10 \mathrm{~dB}, 45^{\circ}\right)\end{array}$ \\
\hline
\end{tabular}

Proof: We first define $\tau_{\mathrm{L}}$ as the probability that there exist LoS mmWave BSs. Similar to (28), the probability that a mobile device selects a LoS mmWave BS for the mmWave energy harvesting is calculated as

$\mathcal{H}_{\mathrm{mm}}^{\mathrm{LoS}}=\tau_{\mathrm{L}} \mathbb{E}_{\left|Y_{o}\right|}[\underbrace{\operatorname{Pr}\left(\Lambda_{\mathrm{mm}_{1}}^{\mathrm{LoS}}>\Lambda_{\mu_{1}}\right)}_{\Theta_{\mu}\left(\left|Y_{o}\right|\right)} \times$

$\underbrace{\operatorname{Pr}\left(\Lambda_{\mathrm{mm}}^{\mathrm{LoS}}>\eta_{\mathrm{mm}} P_{\mathrm{mm}} \mathrm{M}_{\mathrm{B}} \mathrm{M}_{\mathrm{D}} \beta_{\mathrm{NLoS}}^{\mathrm{mm}}\left(R_{\ell}^{\mathrm{mm}}\right)^{-\alpha_{\mathrm{NLoS}}^{\mathrm{mm}}} \mid \ell \in \Phi_{\mathrm{mm}}^{\mathrm{NLoS}}\right)}_{\bar{\Theta}_{\mathrm{N}}\left(\left|Y_{o}\right|\right)}]$

$=\tau_{\mathrm{L}} \int_{0}^{\infty} \Theta_{\mu}(r) \bar{\Theta}_{\mathrm{N}}(r) f_{\left|Y_{o}\right|}(r) d r$,

where $\Lambda_{\mathrm{mm}_{1}}^{\mathrm{LoS}}=\eta_{\mathrm{mm}} P_{\mathrm{mm}} \mathrm{M}_{\mathrm{B}} \mathrm{M}_{\mathrm{D}} \beta_{\mathrm{LoS}}^{\mathrm{mm}}\left|Y_{o}\right|^{-\alpha_{\mathrm{LoS}}^{\mathrm{mm}}}$ is the directed transferred power from the nearest LoS mmWave BS, and the PDF of $\left|Y_{o}\right|$ is given by [10]

$$
f_{\left|Y_{o}\right|}(r)=\frac{2 \pi \lambda_{\mathrm{mm}}}{\tau_{\mathrm{L}}} r f_{\mathrm{Pr}}(r) e^{-2 \pi \lambda_{\mathrm{mm}} \int_{0}^{r} f_{\mathrm{Pr}}(t) t d t} .
$$

Similar to (29), $\Theta_{\mu}(r)$ is derived as

$$
\begin{aligned}
& \Theta_{\mu}(r)=\operatorname{Pr}\left(\eta_{\mathrm{mm}} P_{\mathrm{mm}} \mathrm{M}_{\mathrm{B}} \mathrm{M}_{\mathrm{D}} \beta_{\mathrm{LoS}}^{\mathrm{mm}} r^{-\alpha_{\mathrm{LoS}}^{\mathrm{mm}}}>\eta_{\mu} P_{\mu} N \beta_{\mu} R_{\mu}^{-\alpha_{\mu}}\right) \\
& =\operatorname{Pr}\left(R_{\mu}>\left(\frac{\beta_{\mu}}{\varpi \beta_{\mathrm{LoS}}^{\mathrm{mm}}}\right)^{1 / \alpha_{\mu}} r^{\frac{\alpha_{\mathrm{LoS}}^{\mathrm{mm}}}{\alpha_{\mu}}}\right) \\
& =\exp \left(-\lambda_{\mu} \widetilde{A}_{\mu}(r)\right)
\end{aligned}
$$

where $\widetilde{A}_{\mu}(r)=\pi\left(\frac{\beta_{\mu}}{\varpi \beta_{\mathrm{LoS}}^{\operatorname{mm}}}\right)^{2 / \alpha_{\mu}} r^{\frac{2 \alpha_{\mathrm{LoS}}^{\mathrm{mm}}}{\alpha_{\mu}}}$. Then, $\bar{\Theta}_{\mathrm{N}}(r)$ is similarly derived as

$\bar{\Theta}_{\mathrm{N}}(r)=\exp \left(-2 \pi \lambda_{\mathrm{mm}} \int_{0}^{\widetilde{R}_{\mathrm{NLoS}}^{\mathrm{mm}}(r)}\left(1-f_{\mathrm{Pr}}(t)\right) t d t\right)$,

where $\widetilde{R}_{\mathrm{NLoS}}^{\operatorname{mm}}(r)=\left(\frac{\beta_{\mathrm{NLoS}}^{\operatorname{mm}}}{\beta_{\mathrm{LoS}}^{\operatorname{mos}}}\right)^{1 / \alpha_{\mathrm{NLoS}}^{\operatorname{mm}}} r^{\frac{\alpha_{\mathrm{LoS}}^{\operatorname{mm}}}{\alpha_{\mathrm{NLOS}}^{\mathrm{mLS}}}}$. Substituting (34) and (35) into (32), we obtain (31) and complete the proof.

\section{Simulation Results}

In this section, we present numerical results to show the energy coverage in sub- $6 \mathrm{GHz}$ and mmWave tiers. We assume that the LoS probability function is $f_{\operatorname{Pr}}(R)=e^{-\varrho R}$ with $1 / \varrho=141.4 \mathrm{~m}$ [10]. The basic simulation parameters are shown in Table I.

Fig 1. shows the directed transferred energy coverage probability in sub-6 GHz and mmWave tiers. The analytical results are obtained from (9) and (10), respectively, which are validated by Monte Carlo simulations. The result in (9) 


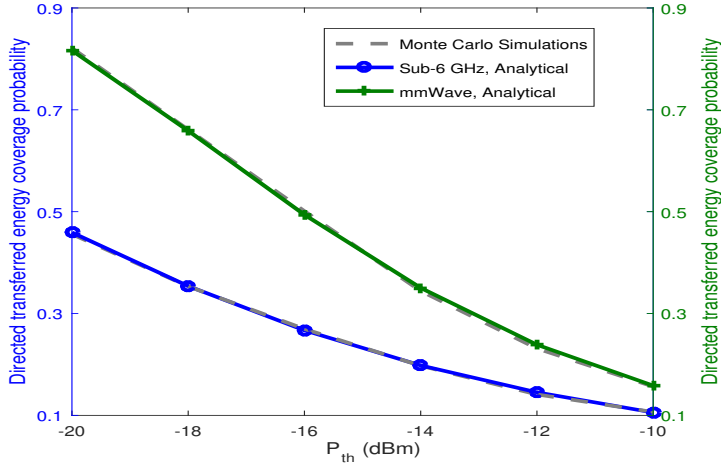

Fig. 1. Directed transferred energy coverage probability with $N=32$, $\alpha_{\mu}=2.7, \lambda_{\mu}=0.002$ and $\lambda_{\mathrm{mm}}=0.02$.

can well predict the energy coverage of the sub- $6 \mathrm{GHz}$ tier. MmWave power transfer can be better than the sub- $6 \mathrm{GHz}$, due to the mmWave directivity gain and densification gain.

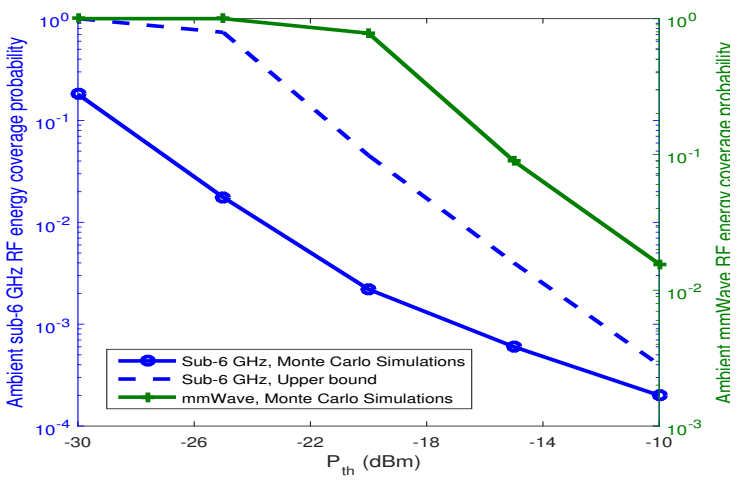

Fig. 2. Ambient RF energy coverage probability with $\alpha_{\mu}=2.6, \lambda_{\mu}=0.002$ and $\lambda_{\mathrm{mm}}=0.5$.

Fig. 2 shows the ambient RF energy coverage probability in sub- $6 \mathrm{GHz}$ and mmWave tiers. We see that for ultra-dense mmWave tier, the ambient mmWave RF energy can still be larger than that in sub-6 GHz tier with comparably lower BS density. Compared to Fig. 1, it is indicated that the amount of harvested energy from ambient RF is much lower than directed power transfer.

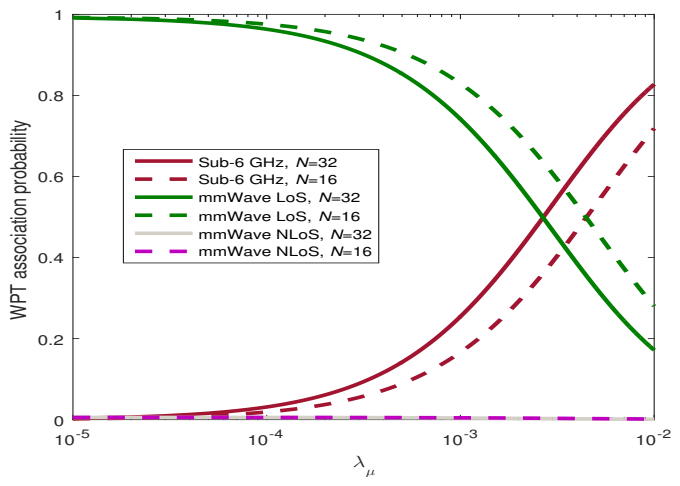

Fig. 3. Power transfer connectivity probability with $\alpha_{\mu}=2.7$ and $\lambda_{\mathrm{mm}}=$ 0.01 .

Fig. 3 shows the power transfer connectivity probability when a MD can select sub-6 GHz or mmWave tier for power transfer in hybrid cellular networks. The results are obtained based on Proposition 1 and Proposition 2. We observe that the sub- $6 \mathrm{GHz}$ power transfer connectivity probability increases with the sub- $6 \mathrm{GHz}$ BS density for obtaining more densification gains, and it will also be improved by adding sub-6 GHz antennas for achieving more antenna gains. More devices will select mmWave BSs to transfer energy when the sub-6 GHz BS density is much smaller than the mmWave BS density, which implies that dense cells are needed to shorten the energy transfer distance between the MD and its associated BS. Compared to the mmWave LoS, the probability that a MD selects a mmWave NLoS power transfer is negligible.

\section{CONCLusions}

In this paper, wireless power transfer was studied in the sub$6 \mathrm{GHz}$ and mmWave dense cellular networks. The expressions for the energy coverage probability in such networks were derived. Comparisons between the sub- $6 \mathrm{GHz}$ and mmWave energy harvesting were provided. In addition, the probability that a mobile device selects the sub- $6 \mathrm{GHz}$ or mmWave power transfer was quantified.

\section{REFERENCES}

[1] K. Huang and X. Zhou, "Cutting the last wires for mobile communications by microwave power transfer," IEEE Commun. Mag., vol. 53, no. 6, pp. 86-93, June 2015 .

[2] G. Yang, C. K. Ho, R. Zhang, and Y. L. Guan, "Throughput optimization for massive MIMO systems powered by wireless energy transfer," IEEE J. Sel. Areas Commun., vol. 33, no. 8, pp. 1640-1650, Aug. 2015.

[3] Y. Zhu, L. Wang, K.-K. Wong, S. Jin, and Z. Zheng, "Wireless power transfer in massive MIMO aided HetNets with user association," IEEE Trans. Commun., Early Access Articles, 1-15, 2016.

[4] L. Wang, M. Elkashlan, R. W. Heath, Jr., M. D. Renzo, and K.-K. Wong, "Millimeter wave power transfer and information transmission," in Proc. IEEE GLOBECOM, San Diego, CA, Dec. 2015, pp. 1-6.

[5] T. A. Khan, A. Alkhateeb, and R. W. Heath, Jr., "Millimeter wave energy harvesting," IEEE Trans. Wireless Commun., pp. 6048-6062, Sep. 2016.

[6] M. D. Renzo and W. Lu, "System-level analysis/optimization of cellular networks with simultaneous wireless information and power transfer: Stochastic geometry modeling," IEEE Trans. Veh. Technol., pp. 1-24, 2016.

[7] T. Rappaport, S. Sun, R. Mayzus, H. Zhao, Y. Azar, K. Wang, G. N. Wong, J. K. Schulz, M. Samimi, and F. Gutierrez, "Millimeter wave mobile communications for $5 \mathrm{G}$ cellular: It will work!" IEEE Access, vol. 1, pp. 335-349, May 2013.

[8] A. M. Hunter, J. G. Andrews, and S. Weber, "Transmission capacity of ad hoc networks with spatial diversity," IEEE Trans. Wireless Commun., vol. 7, no. 12, pp. 5058-5071, Dec. 2008.

[9] L. Wang, H. Q. Ngo, M. Elkashlan, T. Q. Duong, and Kai-Kit Wong, "Massive MIMO in spectrum sharing networks: Achievable rate and power efficiency," IEEE Systems Journal, pp. 1-12, July 2015.

[10] T. Bai and R. W. Heath Jr., "Coverage and rate analysis for millimeterwave cellular networks," IEEE Trans. Wireless Commun., vol. 14, no. 2, pp. 1100-1114, Feb. 2015.

[11] J. Park, S. L. Kim, and J. Zander, "Tractable resource management with uplink decoupled millimeter-wave overlay in ultra-dense cellular networks," IEEE Trans. Wireless Commun., vol. 15, no. 6, pp. 43624379, June 2016.

[12] F. Baccelli, B. Blaszczyszyn, and P. Muhlethaler, "An Aloha protocol for multihop mobile wireless networks," IEEE Trans. Inf. Theory, vol. 52, no. 2, pp. 421-436, Feb. 2006.

[13] K. Huang and V. K. N. Lau, "Enabling wireless power transfer in cellular networks: Architecture, modeling and deployment," IEEE Trans. Wireless Commun., vol. 13, no. 2, pp. 902-912, Feb. 2014.

[14] F. Baccelli and B. Blaszczyszyn, Stochastic Geometry and Wireless Networks, Volume I: Theory. Now Publishers Inc. Hanover, MA, USA, 2009. 